\title{
A CASE OF SCLEROSING MESENTERITIS MIMIC MESENTERIC TUMOUR WITH SMALL BOWEL OBSTRUCTION. A DIFFICULT DIAGNOSIS
}

\author{
Lozev I., G. Kirov, B. Moshev, N. Smilov, D. Dardanov \\ Department of surgery, Medical Institute - Ministry of Interior
}

Reviewed by: prof. R. Madjov

\begin{abstract}
Sclerosing mesenteritis is a rare medical condition, affecting radix mesenterii. Its initial clinical appearance is very atypical. Pathological finding was non-specific and in most cases is associated with inflammatory process. Currently there is no imaging method confirming the diagnosis, which requires in most patients to make laparotomy with biopsy from the changed tissue. At least six months of conservative therapy are needed for the patient to become asymptomatic. The material presents our experience with a patient and brief literature review.
\end{abstract}

Key words: Mesenteritis, Lypodystrophy, Panniculitis, Sclerosing mesenteritis

\section{INTRODUCTION}

Sclerosing mesenteritis is a rarely existent fibrous inflammatory lesions of the infra-abdominal fat tissue with unclear etiology, which usually possesses atypical clinical symptoms. This disease is described as a part of multifocal and systemic fibrosclerosis too, which also includes retroperitoneal fibrosis, sclerosing mediastinitis, thyroiditis of Riedel and orbital pseudotumors. Only 300 patients have been described up to now in the world literature (1). These cases constitute the diversity of symptoms and the difficult for interpretation clinical finding for this suffering. For this reason an extraordinary acumen of the surgeon, radiologist and pathologist is required, because the symptoms can be similar with other diseases calling for completely different therapeutic approach.

The diagnosis is most frequently placed during the time of laparotomy which is done in the case of tumor process with lipomatosis feature in the area of radix mesenterii. The first announcement was made by Jura (2), who describes in 1924 a patient with "contracted sclerosing mesenteritis". This term is again used in 1977 by Durts (3) who describes 68 cases existent in the literature up to this moment and formulates the suffering as benign.

Currently most authors describe this disease as "sclerosing mesenteritis" by dividing it into three stages, each of them constituting a progressively deteriorating condition. Pathological finding starts with fat necrosis and ends with regen-

Address for correspondence:

Ilia Lozev, Medical Institute, Ministry of Interior

79, Skobelev Blvd., Sofia 1606

e-mail: ilialozev@gbg.bg erative fibrosis. During the progression of this suffering fat necrosis dominates the first stage that's why it is usually named mesenteric lypodystrophy. Over the time it is substituted by mesenteric panniculitis which is characterised through intensive inflammatory process and at the end by retractive fibrosing mesenteritis when the fibrosis becomes the main pathological finding.

\section{CASE REPORT}

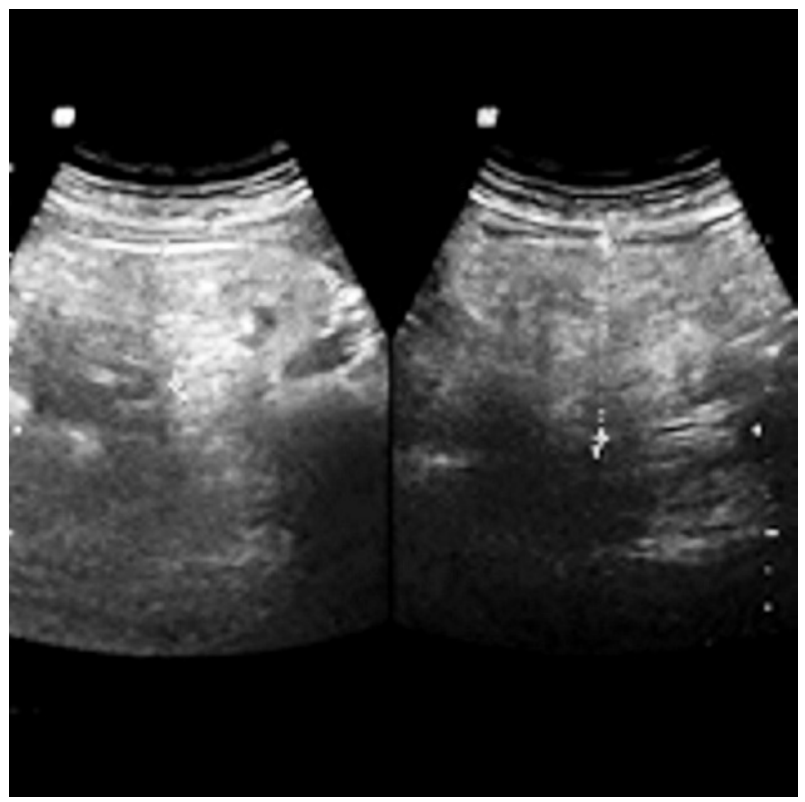

Fig. 1 
A woman at the age of 56 complaining from constant, colic-like pains in the epigastrium and in the left abdominal side, occurring three months before. The patient has reduced $20 \mathrm{~kg}$ in weight. The routine biochemical and hematological examinations are normal. The duplex-doppler check up discovered a relatively moveable with unclear boundaries heterogenic tumor formation echographic structure with presence of doppler signals in it (figure 1). CT shows relatively homogenic, soft-tissue like, hyperdense tumor formation in the areas of radix mesenterii with a diameter of 7 centimeters (figure 2). The boundaries of the formation are unclear from the position of the neighboring small bowel fold which has been almost completely obturated.

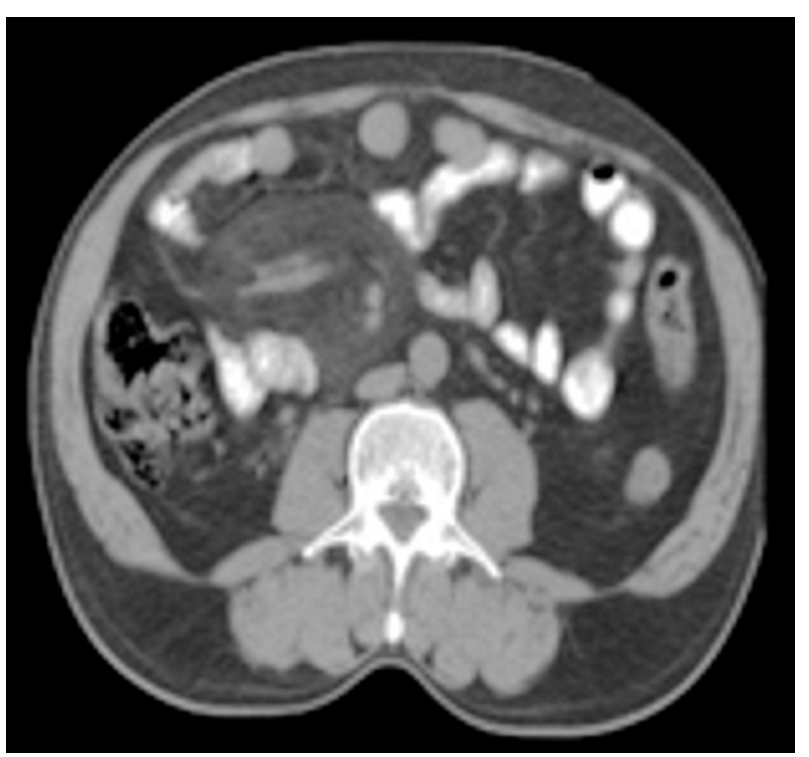

Fig. 2

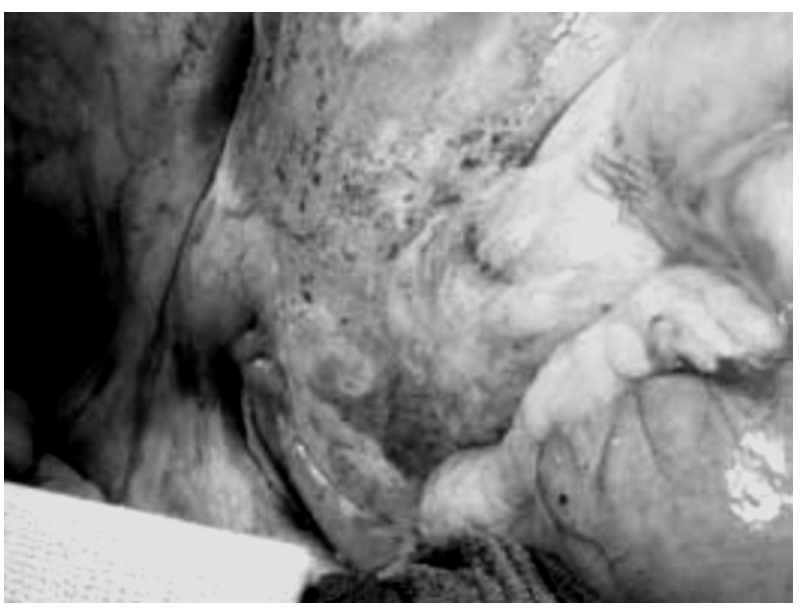

Fig. 3

Under the conditions of delayed urgency the patient is laporotomized in view of removing the tumor formation from radix mesenterii and controlling the ileus-like condition. Intrasurgery found formation in radix mesenterii very close under the lig. Treitz with unclear boundaries, with dimensions 85/60, heavily deforming the adjacent mesenteritis, infiltrating and pressurizing the proximal jejunal fold with pronounced fibrotic changes on its serosa. Express intraoperative histological analysis and the lasting medical substance from the difficult for interpretation formation found inflammatory infiltration, composed of lymphocytes, plasmocytes, histiocytes and eosinophils and multitude of outbreaks, composed of fat necrosis, fibrotic-area proliferation and collagenous fallouts, which excluded neoplastic process (figure 4). In view of overcoming ileus-like condition the formation was removed through resection of the infiltrated small bowel fold. The passage was recovered through termino-terminal anastomosis. The patient has indicated no any substantive complaints during the next 5 months and is with recovered gastrointestinal passage.

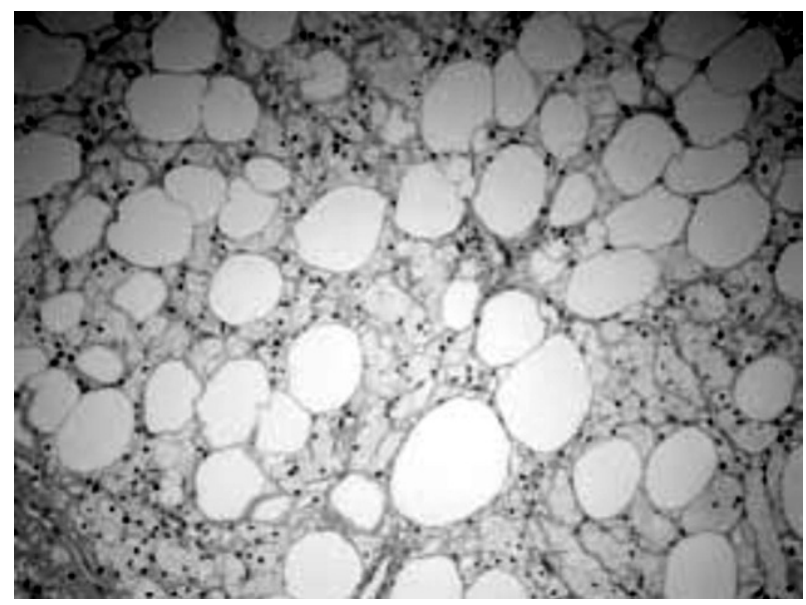

Fig. 4

\section{DISCUSSION}

In the past this rarely observed benign disease is described as mesenteric panniculitis, retractile mesenteritis, isolated lypodystrophy, mesenteric lipogranuloma, engagement of mesenterium from the Weber-Christian disease and so on, but currently is said to be defined as "sclerosing mesenteritis".

Under the three big examinations carried out by Durst (3), Kipfer (5) и Emory (4) 68, 53 and 43 cases were reported accordingly. This disease is still unknown to most surgeons, radiologists and pathologists. It usually affects men (2-3:1) between 50 - 60 years of age (the age varies from 20 to 80 ) by appearing through different clinic profile. For more than half of the cases a formation is found randomly during the time of laparotomy. For the rest of the patients a leading complaint in the clinic profile concerns the difficulty in the gastrointestinal passage (as it is with our patient), expressing itself from postprandial discomfort to acute intestine occlusion. Other rarer clinic expressions can be as follows: febrilitetis, protein-losing enteropathy, intra-abdominal tumor formation with an unclear origin. The biochemical discovery most frequently does not help the diagnosis. CT finding is quite diverse, from the increas- 
ing tissue mesenterium dilution ("unclear radix mesenteritis") to the solid soft-tissue mass with clear boundaries, which can encompass the mesenteric vessels as well, however, leaving one free area of fat tissue around it ("fat ring symptom") (6,7). Usually, no increased regional lymph node knots are found out. Other tumors in the radix mesenterii can express themselves through similar radiological discovery: lipoma, lymphoma, carcinoid tumour, peritoneal carcinosis or tuberculosis, mesothelioma, run-off or hematoma (as a result of cirrhosis, trauma, hypoalbuminemia, heart condition, vasculitis) (8). Percutaneous needle aspiration biopsy or trucat biopsy under an echographic control can help the diagnosis, but because of diagnostic difficulties laparotomy or surgical biopsy is needed for most of the patients. Under the existence of serous diagnostic difficulties following the interpretation of the finding from CT or MRI (as it is in our case), at present some authors $(9,10)$ recommend laparoscopic biopsy. Intra-surgical discovery can be diverse: diffuse enlargement of radix mesenterii, single tumor mass, multitude of tumors, or a sum of nodular hypertrophic creations. Retroperitoneal and pelvic fat tissue, mesocolon and in rarer cases mesoappendix or epiploon are involved for some of the patients. The great diversity of intra-surgical finding is the reason for it to be easily mistaken for many intra-abdominal neoplasms. The histological examination shows different degree of involvement. Lypodystrophy is characterised with infiltration of radix mesenterii from macrophages saturated with fats and greatly reduced healthy fat tissue where under the increase of inflammatory process (panniculitis) lymphocytic infiltration and fat cystic necrosis grow. The last stage is identified by diffuse necrosis and fibrosis which cause tissue retraction observed under retractile mesenteritis. For some patients calcified zones surrounded by multinuclear giant cells are discovered. As for our case macrophages prevail as well as zones of fat necrosis and fibrosis. Staining with hematoxilin and eosin is most frequently sufficient for the precise diagnosis but certain cases require use of immunohistology (11). The deferential diagnosis is made with foreign body-type granuloma (more fibrosis and less lipid-loaded macrophages) and with the disease of Weber-Christian (more frequently affecting the sub-skin tissue and with more lymphocytic infiltration) (12). Lipomatosis, lymphoma, retroperitoneal fibrosis have basically identical microscopic features, but lack fat necrosis (13). Despite the unclear ethiological reason, disease is described by some authors through inter-relationship with other sufferings. For some patients with sclerosing mesenteritis, AIDS (14), non-Hodgkin lymphoma (15), tuberculosis (1) or cholesterolic fallouts in the mesenterium (16) are to be found.

Conservative therapy has not been standardised yet and is dependent on the stage of the illness. During the first stage, when fat necrosis is dominating, most authors recommend awaiting tactics because there is a tendency towards spontaneous regression. The chronic inflammation requires corticosteroid therapy and various types of immunosuppression. Positive results are reported by cyclophosphamide, colchicine, azathioprine and by oral progesteron (17). An intestinal obstruction can be developed in intensive fibrosis which will require intestinal resection bypass or colostoma formation $(18,19)$.

\section{CONCLUSION}

The diagnosis of this non-specific, benign, inflammatory disease is a serious challenge for radiologists, surgeons and pathologists. Some authors (20) mention that the number of affected patients is much larger in comparison with the reported cases. Among the difficulties with placing our case diagnosis is in the consideration that in cases of great tumor formations in patients with obesity, laparotomy is necessary in order to reach final diagnosis or at least macro-biopsy of the tumor mass because express intraoperative histological examination and imaging findings often are contradictory.

\section{REFERENCES}

1. Ege G, Akman H, Cakiroglu G. Mesenteric panniculitis associated with abdominal tuberculous lymphadenitis: a case report and review of the literature. Br J Radiol 2002; 75: 378-380

2. Jura V. Mesenterite retrattile-caso clinico: risultati sperimentali, rilievi patogenetici, considerazoni cliniche. Policlinico (sez. Chir) 1927; 34: 535-556, 566-599

3. Durst AL, Freund H, Rosenmann E, Birnbaum D. Mesenteric panniculitis: review of the leterature and presentation of cases. Surgery 1977; 81: 203-211

4. Emory TS, Monihan JM, Carr NJ, Sobin LH. Sclerosing mesenteritis, mesenteric panniculitis and mesenteric lipodystrophy: a single entity? Am J Surg Pathol 1997; 21: 392-398

5. Kipfer RE, Moertel CG, Dahlin DC. Mesenteric lipodystrophy. Ann Intern Med 1974; 80: 582-588

6. Horton KM, Lawler LP, Fishman EK. CT findings in sclerosing mesenteritis (panniculitis): spectrum of disease. Radiographics 2003; 23: 1561-1567

7. Patel N, Saleeb SF, Teplick SK. General case of the day. Mesenteric panniculitis with extensive inflammatory involvement of the peritoneum and intraperitoneal structures. Radiographics 1999; 19: 1083-1085

8. Seo BK, Ha HK, Kim AY, Kim TK, Kim MJ, Byun JH, Kim PN, Lee MG, Yang SK, Yu ES, Kim JH. Segmental misty mesentery: analysis of CT features and primary causes. Radiology 2003; 226: 86-94

9. Weiser J, Salky B, Slepian A, Dikman S. Laparoscopic diagnosis of retractile mesenteritis. Gastrointest Endosc 1992; 38: 615-617

10. Rajendran B, Duerksen DR. Retractile mesenteritis presenting as protein-losing gastroenteropathy. Can J Gastroenterol 2006; 20: 787-789 
11. Montgomery E, Torbenson MS, Kaushal M, Fisher C, Abraham SC. Beta-catenin immunohistochemistry separates mesenteric fibromatosis from gastrointestinal stromal tumor and sclerosing mesenteritis. Am J Surg Pathol 2002; 26: 1296-1301

12. Wang H, Recant W, Montag AG, Hart J. Pathologic quiz case. A large mesentric mass in 40-year-old man. Arch Pathol Lab Med 2001; 125 : 443-444

13. Bashir MS, Abbott CR. Mesenteric lipodystrophy. J Clin Pathol 1993; 46: 872-874

14. Aboulafia DM. Inflammatory pseudotumor causing small bowel obstruction and mimicking lymphoma in a patient with AIDS: clinical improvement after initiation of thalidomide treatment. Clin Infect Dis 2000; 30: $826-831$

15. Petrovic D, Jovanovic D, Guduric B, Filipovic B, Mutibaric A. Primary non-Hodgkin lymphoma of the jejunum associated with mesenteric lipodystrophy: a case report. Arch Oncol 2004; 12 : $67-70$
16. Cherayil GD, Scaria KS, Hensley GT, Elliott WH. Abnormal lipid composition of fat tissue in human mesenteric panniculitis. Lipids 1981; 16: 199-202

17. Colomer Rubio E, Blanes Gallego A, Carbonell Biot C, Villar Grimalt A, Tomas Ivorra H, Llamusi Lorente A. Mesenteric panniculitis with retroperitoneal involvement resolved after treatment with intravenous cyclophosphamide pulses. An Med Interna 2003; 20: 31-33

18. Shah DM, Patel SB, Shah SR, Goswami KG. Mesenteric panniculitis a case report and review of literature. Ind J Radiol Imag 2005; 15: 191-192

19. Parra-Davila E, McKenney MG, Sleeman D, Hartmann R, Rao RK, McKenney K, Compton RP. Mesenteric panniculitis: case report and literature review. Am Surg 1998; 64: 768-771

20. Khachaturian T, Hughes J. Mesenteric panniculitis. West J Med 1988; 148: 700-701

21. Jaime Poniachik T, Gladys Smok S. Mesenterits retractil. Com unicacion de cuat ro casos. Rev Med Chile 2000; 128(11). 